\section{Monitoring plan for the detection of gluten not declared on labels in meat preparations: preliminary results}

\author{
Maria Concetta Campagna, ${ }^{1}$ \\ Antonella Nardoni, ${ }^{1}$ \\ Francesca Della Verità, ${ }^{1}$ Selene Marozzi, ${ }^{1}$ \\ Rita Marcianò, ${ }^{2}$ Roberto Condoleo' \\ 'Istituto Zooprofilattico Sperimentale \\ delle Regioni Lazio e Toscana, Roma; \\ ${ }^{2}$ Regione Lazio, Area Sanità Veterinaria, \\ Roma, Italy
}

\section{Abstract}

European Union (EU) legislation protects consumers who have a known sensitivity to gluten making obligatory to label the presence of this protein in all foods. Lazio region has therefore adopted a four-year monitoring plan in order to acquire information about the presence of undeclared gluten in products considered as a serious risk to food safety. Preliminary data reported in this work indicate the absence of gluten in all 100 samples analyzed ( 29 meat preparations and 71 meat products), in accordance with legislation in force. However, the number of samples of this work is still limited and therefore additional data need to be collected.

\section{Introduzione}

La celiachia, detta anche malattia celiaca, è una patologia autoimmune attivata dall'ingestione della gliadina, una delle componenti del glutine (Mowat, 2003; Sollid, 2002). Quest'ultimo è una frazione proteica insolubile presente nel frumento (o grano), nell'orzo, nella segale, nell'avena, nel farro e nel kamut. Qualsiasi alimento che deriva da tali cereali, o è contaminato accidentalmente da questi in qualsiasi fase della filiera alimentare, risulta dannoso per gli individui affetti dalla malattia. In Europa, Nord e Sud America, Nord Africa e continente Sub-Indiano, si stima che l'incidenza della celiachia, nella popolazione, sia approssimativamente dell'1\% (Hischenhuber et al., 2006).

Al fine di tutelare tale fascia di consumatori a rischio aventi sensibilità nota nei confronti del glutine, la Commissione Europea ha emanato la Direttiva CE/89/2003, secondo la quale è obbligatorio indicare in etichetta la presenza di tale proteina qualora questa sia stata impiegata come ingrediente (Commissione
Europea, 2003; Repubblica Italiana, 2006). Il Regolamento CE 41/2009 ha successivamente introdotto ulteriori opportunità in materia di etichettatura, ovvero la possibilità di recare la dicitura senza glutine qualora l'azienda produttrice sia in grado di garantire che la sostanza non è presente in quantità superiore a 20 $\mathrm{mg} / \mathrm{kg} \quad$ (Repubblica Italiana, 2009; Commissione Europea, 2009).

Nonostante tali normative, i dati forniti dal Rapid Allert System for Food and Feed (RASFF) riportano 58 notifiche riguardanti la presenza non dichiarata di glutine in prodotti alimentari in fase di commercializzazione (dal 1 gennaio 2008 al 31 Dicembre 2012), di cui 6 (10\%) riguardanti preparazioni di carne (DG SANCO, 2012). Il ritrovamento del glutine in questi alimenti può essere dovuto all'impiego volontario di tale proteina per motivazioni tecnologiche. Proteine di origine non animale, come quelle derivanti dai cereali, sono infatti spesso utilizzate come agenti gelificanti alternativi in prodotti a base di carni macinate perché, grazie alla loro proprietà di legare l'acqua presente, permettono di aumentare la resa e la consistenza dell'alimento (Pietrasik et al., 2007). La presenza indesiderata di glutine può essere inoltre legata a cross-contaminazioni in fase di produzione 0 durante le fasi di commercializzazione e vendita.

Nell'ambito della programmazione regionale dei controlli ufficiali, la Regione Lazio ha quindi previsto un piano di monitoraggio quadriennale proprio al fine di acquisire informazioni riguardo la presenza non dichiarata di tale allergene in alimenti ritenuti a rischio, quali le preparazioni a base di carne. I dati raccolti saranno utilizzati per valutare il rischio connesso al consumo di queste tipologie alimentari anche in funzione dei diversi fattori ritenuti rilevanti. Il presente studio riporta sinteticamente i primi dati ottenuti dalle attività previste dal piano.

\section{Materiali e Metodi}

Dal Marzo 2011 al Giugno 2012, i servizi veterinari della Regione Lazio hanno prelevato, nell'ambito del piano regionale dei controlli, 100 campioni di preparazioni (n. 29) e prodotti (n. 71) a base di carne in esercizi di vendita al dettaglio. I campioni sono stati prelevati in singola aliquota e sono stati conferiti all'Istituto Zooprofilattico Sperimentale della Regione Lazio dove sono stati analizzati con tecnica immunoenzimatica (ELISA) mediante Gluten Assay Kit della ditta BioKits (Dublin, $\mathrm{OH}, \mathrm{USA})$ [limit of detection (LOD)=1 ppm]. Tale metodo prevede una preventiva omogeneizzazione del campione, seguita da estrazione con uno specifico tampone. Dopo incubazione, centrifugazione e filtrazione l'estratto è
Correspondence: Roberto Condoleo, Istituto Zooprofilattico Sperimentale delle Regioni Lazio e Toscana, via Appia Nuova 1411, 00178 Roma, Italy.

Tel. +39.067099360 - Fax: +39.067099312 .

E-mail: roberto.condoleo@izslt.it

Key words: Allergen, Meat products, Gluten.

Received for publication: 15 January 2013.

Revision received: 18 March 2013.

Accepted for publication: 19 March 2013

This work is licensed under a Creative Commons Attribution 3.0 License (by-nc 3.0).

(O) Copyright M.C. Campagna et al., 2013

Licensee PAGEPress, Italy

Italian Journal of Food Safety 2013; 2:e15

doi:10.4081/ijfs.2013.e15

stato sottoposto a saggio immunoenzimatico, utilizzando micropiastre dotate di micropozzetti rivestiti con anticorpi monoclonali antigliadina in grado di legarsi con il glutine eventualmente presente. Dopo l'eliminazione della frazione non legata tramite lavaggi, è stato aggiunto un secondo anticorpo anti-gliadina coniugato con la peroxidase che, in caso di presenza di glutine, si lega all'immunocomplesso primario formando un sandwich. Successivamente è stata quindi aggiunta della tetrametilbenzidina (TMB), un substrato in grado di rilevare la presenza di glutine, mediante un viraggio di colore direttamente proporzionale alla concentrazione di proteina presente.

\section{Risultati}

Come riportato nella Tabella 1, in nessuno dei 100 campioni pervenuti (29 preparazioni di carne e 71 prodotti a base di carne) è stata rilevata la presenza di glutine in concentrazioni superiori al limite di rilevabilità del metodo [massima prevalenza possibile 2,95\%; confidence level (CL) 95\%].

\section{Discussione}

I risultati riportati, seppure preliminari, indicano che i prodotti erano conformi alla normativa in vigore in materia di allergeni 0 comunque permetterebbero di ipotizzare una bassa prevalenza di preparazioni di carne 0 prodotti a base di carne contenenti glutine non dichiarato in etichetta. Non si hanno informazioni riguardo la possibile presenza nelle aziende produttrici degli alimenti prelevati di 
Tabella 1. Numero di campioni analizzati distinti per categoria e tipologia di prodotto.

\begin{tabular}{|c|c|c|c|c|}
\hline \multirow[t]{2}{*}{ Categoria alimentare } & \multirow[t]{2}{*}{ Tipologia prodotto } & \multicolumn{2}{|c|}{ Campioni } & \multirow{2}{*}{$\begin{array}{l}\text { Presenza di } \\
\text { glutine }\end{array}$} \\
\hline & & n & $\%$ & \\
\hline \multirow[t]{6}{*}{ Preparazioni di carne } & Hamburger & 10 & 10,0 & - \\
\hline & Salsicce & 8 & 8,0 & - \\
\hline & Altro & 5 & 5,0 & - \\
\hline & Spiedini & 2 & 2,0 & - \\
\hline & Polpette & 2 & 2,0 & - \\
\hline & Petto di tacchino & 2 & 2,0 & - \\
\hline \multirow[t]{8}{*}{ Prodotti a base di carne } & Wurstel & 17 & 17,0 & - \\
\hline & Prosciutto & 15 & 15,0 & - \\
\hline & Mortadella & 15 & 15,0 & - \\
\hline & Salame & 11 & 11,0 & - \\
\hline & Arrosti & 5 & 5,0 & - \\
\hline & Altro & 4 & 4,0 & - \\
\hline & Zampone & 2 & 2,0 & - \\
\hline & Pancetta & 2 & 2,0 & - \\
\hline Totale & & 100 & 100,0 & \\
\hline
\end{tabular}

altre linee produttive che facciano uso di ingredienti contenenti glutine. Tuttavia, come definito dal piano di monitoraggio, tutti i prodotti confezionati sottoposti al campionamento non recavano in etichetta alcuna dizione cautelativa del tipo può contenere traccie di... 0 prodotto in uno stabilimento che utilizza ingredienti contenenti glutine. Ciò lascia presupporre l'assenza di altre lavorazioni all'interno dello stabilimento in grado di causare potenziali cross-contaminazioni o comunque che il produttore abbia ritenuto improbabile tale evenienza.

Il confronto dei dati ottenuti con altri studi relativamente a tale matrice risulta difficile in considerazione della scarsità di lavori presenti in letteratura riguardanti i prodotti carnei contaminati da glutine. I pochi studi di prevalenza paragonabili al presente lavoro riportano infatti dati su una vasta gamma di prodotti e solo poche osservazioni sono relative ai prodotti carnei (Gregorek et al., 2006; Surojanametakul et al., 2012).

La presenza di notifiche diramate dal RASFF riguardanti prodotti carnei è comunque motivo di interesse per la prosecuzione delle indagini perché induce a supporre un rischio potenziale connesso al consumo di questa categoria di prodotti. Infatti, la presenza non dichiarata di glutine nei prodotti oggetto dello studio è in grado di provocare danni molto seri alla salute dei consumatori sensibili alla proteina. Le con- centrazioni necessarie per scatenare una reazione avversa sono inoltre molto basse per alcuni individui a rischio e quindi sono sufficienti anche contaminazioni modeste per rendere il prodotto dannoso per la salute umana (Hischenhuber et al., 2006).

\section{Conclusioni}

In conclusione, il numero dei campioni esare la casistica per avere un quadro maggiormente esaustivo. Al termine del piano, un maggior numero di osservazioni permetterà inoltre di indagare alcune variabili di interesse come la modalità di vendita, la presenza 0 meno di etichettatura specifica riguardante l'assenza di glutine, e la tipologia specifica di prodotto.

\section{Bibliografia}

Commissione Europea, 2003. Direttiva del Parlamento Europeo del 10 novembre 2003 che modifica la direttiva 2000/13/CE per quanto riguarda l'indicazione degli ingredienti contenuti nei prodotti alimentari, 2003/89/CE. In: Gazzetta Ufficiale, L 308/15, 25/11/2003. minati è limitato, per cui è necessario amplia-
Commissione Europea, 2009. Regolamento della Commissione del 20 gennaio 2009 relativo alla composizione e all'etichettatura dei prodotti alimentari adatti alle persone intolleranti al glutine, 41/2009/CE. In: Gazzetta Ufficiale, L 16/3, 21/01/2009.

DG SANC0, 2012. RASFF Portal 2012. Directorate-General for Health and Consumers, European Commission ed., Brussels, Belgium. Disponibile al sito: https://webgate.ec.europa.eu/rasffwindow/portal/ Ultimo accesso: 26/06/2012.

Gregorek H, Stolarczyk A, Socha J, 2006. Residual gluten contamination in some foodstuffs used in gluten-free diet by coeliac patients in Poland. Pol J Food Nutr Sci 15:329-32.

Hischenhuber C, Crevel R, Jarry B, Maki M, Moneret-Vautrin DA, Romano A, Troncone R, Ward R, 2006. Safe amounts of gluten for patients with wheat allergy or coeliac disease. Aliment Pharm Ther 23:559-75.

Mowat AM, 2003. Coeliac disease: a meeting point for genetics, immunology, and protein chemistry. Lancet 361:1290-92.

Pietrasik Z, Jarmoluk A, Shand PJ, 2007. Effect of non-meat proteins on hydration and textural properties of pork meat gels enhanced with microbial transglutaminase. LWT-Food Sci Technol 40:915-20.

Repubblica Italiana, 2006. Attuazione delle direttive 2003/89/CE, 2004/77/CE e 2005/63/CE in materia di indicazione degli ingredienti contenuti nei prodotti alimentari, DL 114/2006. In: Gazzetta Ufficiale no. 69, 23/03/2006.

Repubblica Italiana, 2009. Circolare del Ministero del lavoro, della salute e delle politiche sociali - 5 Novembre 2009. Linee di demarcazione tra integratori alimentari, prodotti destinati ad una alimentazione particolare e alimenti addizionati di vitamine e minerali: criteri di composizione e di etichettatura di alcune categorie di prodotti destinati ad una alimentazione particolare (09A14198). In: Gazzetta Ufficiale no. 277, $27 / 11 / 2009$.

Sollid LM, 2002. Coeliac disease: dissecting a complex inflammatory disorder. Nat Rev Immunol 2:647-55.

Surojanametakul V, Khaiprapai P, Jithan P, Varanyanond $\mathrm{W}$, Shojib M, Ito T, Tamura $\mathrm{H}$, 2012. Investigation of undeclared food allergens in commercial Thai food products. Food Control 23:1-6. 